\title{
APPLICATION OF HUMAN CENTRIFUGE TO SIMULATE PARABOLIC FLIGHT: EARLY EXPERIENCE
}

\author{
Krzysztof KOWALCZUK', Stefan GAŹDZIŃSKI' ${ }^{2}$ Mariusz WALAS', Marcin STROJEK'1, \\ Michał JANEWICZ'1 , Tadeusz GRZESZUK'1, Kjetil WORMNES ${ }^{3}$, Andreas WERNER ${ }^{4,5}$ \\ 1 Department of Simulator Studies and Aeromedical Training, Military Institute of Aviation Medicine, \\ Warsaw, Poland \\ 2 Creative Neuroscience Lab - CNS Lab, Military Institute of Aviation Medicine, Warsaw, Poland \\ 3 European Space Agency, Noordwijk, Netherlands \\ 4 German Air Force - Centre of Aerospace Medicine - Aviation Physiology Diagnostics and Research, \\ Königsbrück, Germany \\ 5 University Medicine Berlin, Charité Berlin - Campus Charité Mitte (CCM/CCO), Institute for Physiology \& \\ Center of Space Medicine and extreme environments, Berlin, Germany
}

Source of support: Own sources

Author's address: K. Kowalczuk, Military Institute of Aviation Medicine, Krasińskiego 54/56 Street, 01-755 Warsaw, Poland, e-mail: kkowalczuk@wiml.waw.pl

Introduction: We have used our human centrifuge to simulate the parabolic flight environment. Tests were carried out on four candidates taking part in a parabolic flight program. The main idea behind the project was to test the possibility of simulation of a near zero Gz environment with the centrifuge as a cheaper and simpler alternative to parabolic flight. An additional goal was to try to identify crew members who would present excessive symptoms of motion sickness.

Methods: The flight profile was adjusted to replicate the characteristic conditions and specificity of a parabolic flight. The study was performed as a practical test in a variable acceleration environment similar to a real parabolic flight (with G-limits: max. $+2 \mathrm{Gz}$, $\min .0 \mathrm{Gz}$, and up to 13 repetitions of a cycle). Evaluation of eye-hand coordination, orientation and Romberg tests were performed. Each candidate was monitored (HR, ECG, SaO2, earpulse). The exposure was performed twice on two consecutive days.

Results: All participants completed the expositions with positive outcomes. They reported that they had the illusion of microgravity. They exhibited similar hand-eye coordination issues as in microgravity. Last but not least, with repeated exposures, the average heart rates were decreasing, pointing to adaptation to the procedure. Trained behaviors and organism reactions have been successfully used and verified afterwards during the real parabolic flight organized by the ESA. 
Conclusions: Despite the preliminary nature of the study, promising results were obtained, which may be further developed and ultimately used to improve the quality and effectiveness, especially the cost effectiveness, of ground preparation of astronauts for microgravity. Furthermore, the profile might be adjusted to evaluate experiments involving the use of neuroimaging techniques in space.

Keywords: human centrifuge, parabolic flight, micro-gravity, simulation, adaptation

\section{INTRODUCTION}

The parabolic flight (PF) is considered to be an analog of microgravity $(\mu-g)$ that is widely used in mimicking some of the features of spaceflight in a more-controlled and less-expensive manner. In $\mathrm{PF}$, an aircraft flies in sequences of parabolic trajectories that provide short-duration periods of free fall ( $\mu-\mathrm{g}$ for at least 20 to $23 \mathrm{sec}$, see fig. 1). Before and after the $\mu$-g phase the aircraft has a mild high positive G-force (posGz) pull-up and pull-out (1.5 to $1.8 \mathrm{~g}, 20 \mathrm{sec}$ ). Between each parabola, there has been a "recovery phase" (30 to $40 \mathrm{sec}$ ). The parabolic flight is unique in allowing for real $\mu-g$ in a ground-based facility [4]. However, parabolic flight campaigns (PFC) are still pretty expensive and can only simulate a short time $\mu-\mathrm{g}$.

In the long-arm human centrifuge (HTC) at the Military Institute of Aviation Medicine [1,2,3] an active gondola can be rotated in different axes during centrifugation. This allows for acceleration with positive Gz-forces (posGz) used in military high-posGz training. But it can also generate neg- ative $\mathrm{Gz}$-forces (negGz - by rotating the gondola in the other direction) analog to $\mu-g$ due to the fluid shift component in $\mu-g$. The idea behind this project was to demonstrate problems with precise hand movements in microgravity to the future crew of European Space Agency's (ESA) eDeorbit mission involving $\mu-\mathrm{g}$. It was designed to capture and deorbit large items of space debris as part of the Agency's Clean Space initiative.

Certainly, an HTC is not the same as a PFCs because in one direction gravitation is still 1 and $\mu-\mathrm{g}$ is only the result of gyration in axes. Therefore, the spin and its source of symptoms result in a state similar to motion sickness. The project aimed to select crewmembers for space missions by identifying individuals who would develop excessive symptoms of motion sickness. An additional goal of the training was to assess the eye-hand coordination of the participants, as well as examining how they deal with various psychophysiological factors (such as disorientation, reaction to stimuli,

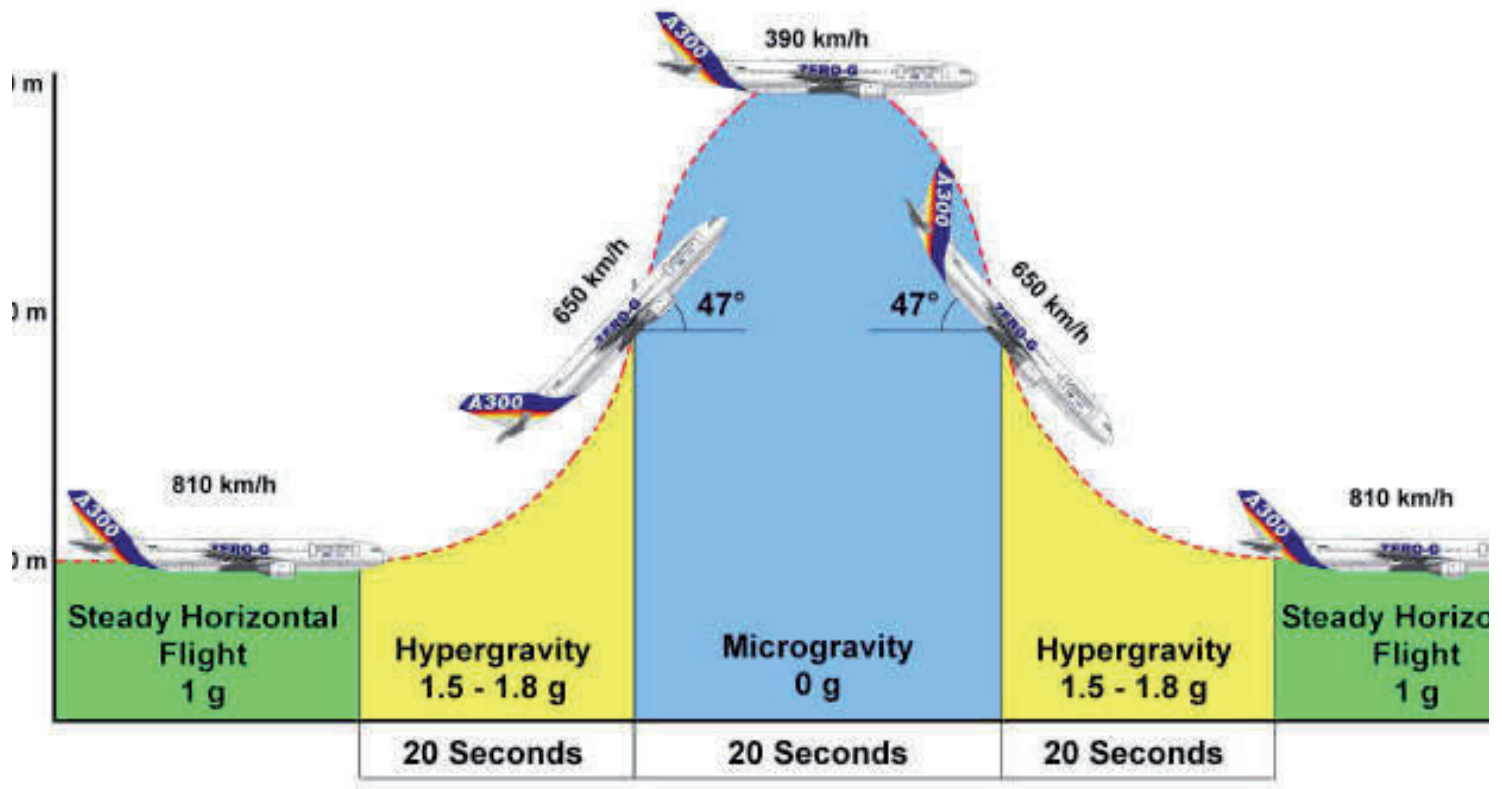

Fig. 1. Accelerations encountered during parabolic flight. (source: https://www.esa.int/spaceinimages/Images/2014/12/ Prabolic_flight_manouvres). At MIAM, only the part shaded in blue was simulated. 
cyclically variable accelerations) that may impact the human body during such exposure. Looking into the future, we expect that this approach could be used as an essential part of the selection, and furthermore - for training before human space flight.

\section{METHODS}

Four representatives of ESA preparing for the unique experience of weightlessness on a PFC participated in the centrifuge training developed by the Aeromedical Training Department of MIAM. Before the HTC training, the participants underwent extensive ENT examinations to eliminate any labyrinth disorders. The substantive scope of the HTC training was focused on maximizing the experience of acceleration and gravitational conditions prevailing during PF.

The acceleration profile was adjusted to replicate similar conditions and PF specificity. The study was performed as a practical test in a variable acceleration environment similar to real PF (with G-limits: max. 2 posGz, min. $\mu-G z$, see fig. 2). The acceleration profile was designed to reflect the z-acceleration profiles and their durations during the parabolic flight. On day one, there were up to seven repetitions of the cycle (acceleration profile), each of which simulated one parabolic flight. After each repetition of the profile, the participants were asked if they wish to continue. If they declined, the exposition was terminated. On the second day, the participants were exposed to up to 13 cycles. The numbers of repetitions were shortened at the pilot's request due to symptoms of motion sickness.

Evaluation of eye-hand coordination, orientation, and Romberg tests were performed. Each candidate was monitored (HR, ECG, SaO2, earpulse). The exposure took place twice on two consecutive days. The mean HRs were calculated over the 20 second-long periods with $\mathrm{Gz}=2.0$ (preceding microgravity) $\mathrm{Gz}=0$ (microgravity), and $\mathrm{Gz}=2.0$ (after microgravity).

The study protocol was approved in advance by the Bioethical Committee of the Military Institute of Aviation Medicine in Warsaw. Each subject provided written informed consent before participation and they were compensated for taking part in the experiment.

\section{RESULTS}

All participants completed the expositions with positive outcomes. The first participant completed 13 cycles on the first day and seven on the second day (fig 4). The other pilots respectively completed 12 and 6 cycles (fig. 5), 13 and 3 cycles (fig 6), and 13 and 5 cycles (fig 7).

They reported that they had the illusion of microgravity. They exhibited similar hand-eye coordination issues as in microgravity (fig. 3).

The transitions between the different phases of the profiles, in particular the switch from $\mu-g$ to $2 \mathrm{G}$, were reported to be the most difficult for the participants. It was when the boogie of the centrifuge flipped upside down while the centrifuge was still rotating, leading to significant symptoms of motion sickness. Another such event was during the centrifuge was braked to stop. For the rest of the time, no problems were reported, unless the participant moved their head.

Another finding was improvement with Romberg tests. Initially the participants had problems with touching their noses with their index fingers (usually they ended up somewhere in the skull

\section{Centrifuge training for parabolic flight}

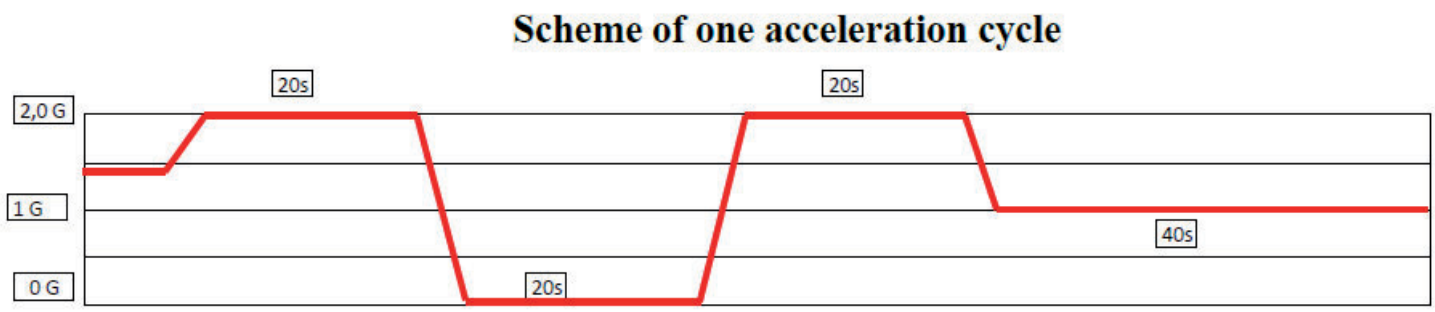

Fig. 2. The timing of z-accelerations during one cycle (single parabolic flight). This cycle (single parabolic flight) was repeated up to 13 times on the first day and up to seven times on the second day of training. 

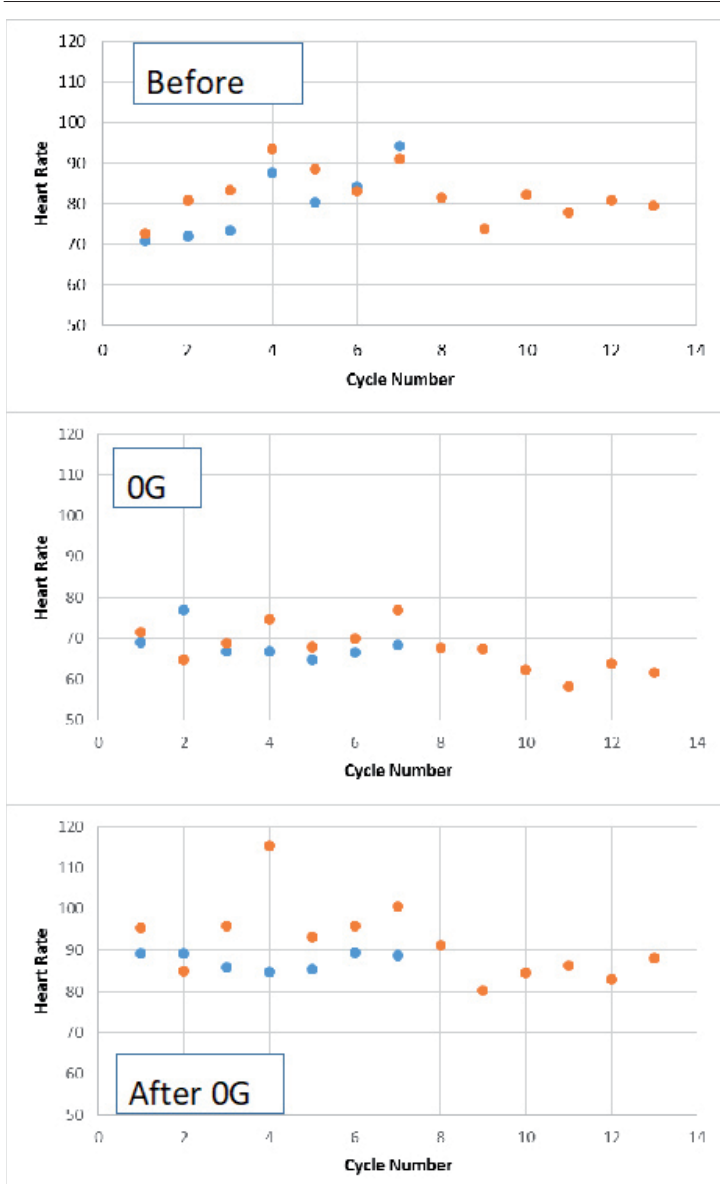

Fig. 4. Changes in average heart rate in consecutive cycles preceding simulated zero gravity, during simulated zero gravity, and afterwards (participant \#1). Blue dots = 1st day; red dots = 2nd day. "Cycle number" refers to the successive number of the flight profile depicted in fig. 2.

frontal area). After couple of repetitions this function went back to normal after a short time.

Last but not least, with repeated exposures, the average heart rates decreased, indicating adaptation to the procedure (fig. 4, 5, 6, 7).

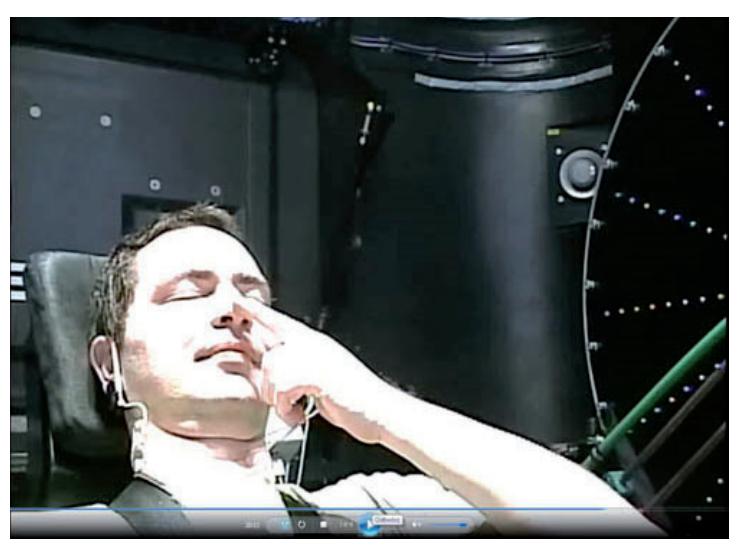

Fig. 3. A participant performing the Romberg test during simulated microgravity.
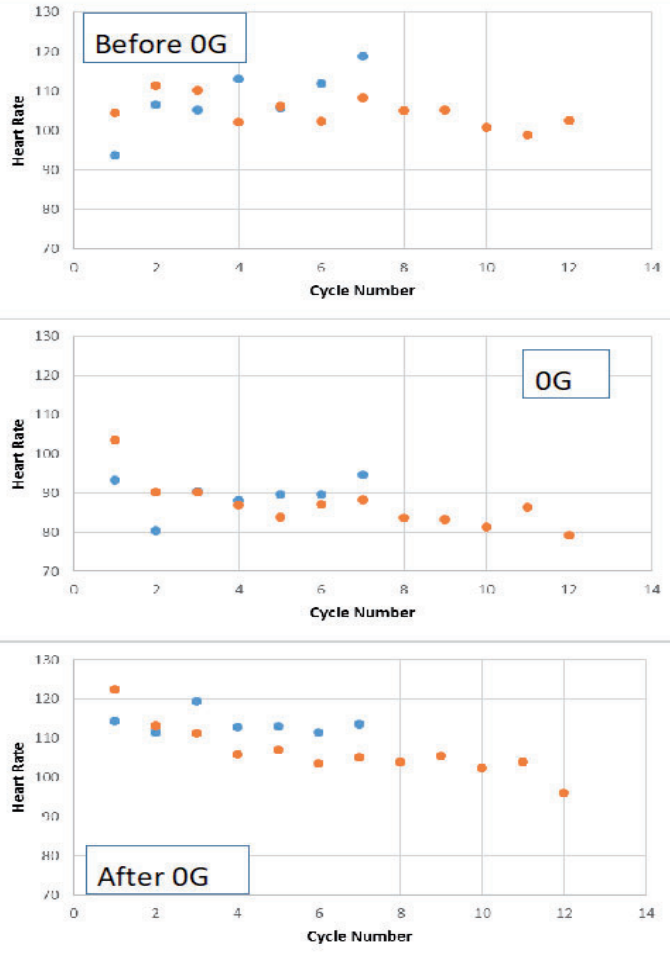

Fig. 5. Changes in average heart rate in consecutive cycles preceding simulated zero gravity, during simulated zero gravity, and afterwards (participant \#2). Blue dots = 1st day; red dots = 2nd day. "Cycle number" refers to the successive number of the flight profile depicted in fig. 2 .

\section{DISCUSSION}

Trained behaviors and organism reactions have afterwards been successfully used and verified during the real parabolic flight organized by the ESA. Despite the preliminary nature of the study, promising results were obtained, which may be further developed and ultimately used for improving the quality and effectiveness, especially the cost-effectiveness, of ground preparation of astronauts for microgravity.

\section{ACKNOWLEDGMENTS}

This study was shortly described at http://www. esa.int/spaceinimages/Images/2015/02/Polish_ human_centrifuge and at http://www.wiml.waw. $\mathrm{pl} / ? \mathrm{q}=\mathrm{en} /$ node/478. 

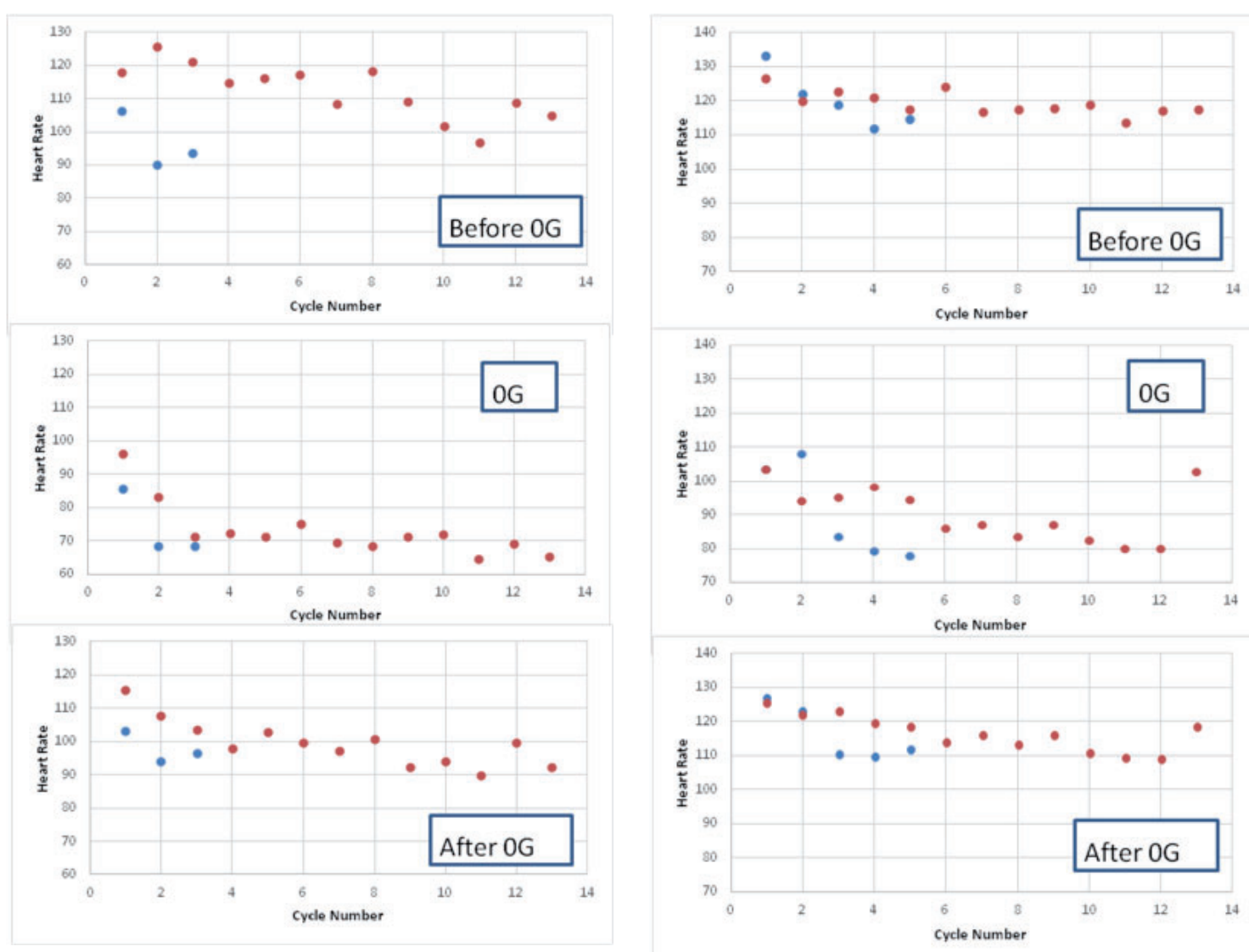

Fig. 6. Changes in average heart rate in consecutive cycles preceding simulated zero gravity, during simulated zero gravity, and afterwards (participant \#3). Blue dots = 1st day; red dots = 2nd day. "Cycle number" refers to the successive number of the flight profile depicted in fig. 2.

Fig. 7. Changes in average heart rate in consecutive cycles preceding simulated zero gravity, during simulated zero gravity, and afterwards (participant \#4). Blue dots = 1st day; red dots = 2nd day. "Cycle number" refers to the successive number of the flight profile depicted in fig. 2.

\section{AUTHORS' DECLARATION:}

Study Design: Krzysztof Kowalczuk, Kjetil Wormnes, Mariusz Walas, Marcin Strojek, Tadeusz Grzeszuk. Data Collection: Michał Janewicz, Mariusz Walas, Marcin Strojek, Tadeusz Grzeszuk. Manuscript preparation: Krzysztof Kowalczuk, Stefan Gazdzinski, Andreas Werner. Funds Collection: Kjetil Wormnes. The Authors declare that there is no conflict of interest.

\section{REFERENCES}

1. Biernacki MP, Tarnowski A, Lengsfeld K, Lewkowicz R, Kowalczuk K, Deren M. +G(z) Load and Executive Functions. Aviation Space and Environmental Medicine. 2013; 84(5):511-5.

2. http://www.wiml.waw.pl/?q=en/Dynamic_Flight_Simulator

3. Kowalczuk K, Puchalska L, Sobotnicki A, Czerw M, Janewicz M, Wylezol M, et al. Effects of Gradual Onset + Gz on Hemodynamic Parameters and Brain Oxygenation in Military Pilots: Preliminary Study. The Polish Journal of Aviation Medicine, Bioengineering and Psychology. 2016; 22(3):5-11.

4. Shelhamer M. Parabolic flight as a spaceflight analog. Journal of Applied Physiology. 2016; 120(12):1442-8.

Cite this article as: Kowalczuk K, Gaździński S, Walas M, Strojek M, Janewicz M, Grzeszuk T, Wormnes K, Werner A. Application of Human Centrifuge to Simulate Parabolic Flight: Early Experience. Pol J Aviat Med Bioeng Psychol 2018; 24(2): 21-25. DOI: 10.13174/ pjambp.30.10.2019.03 